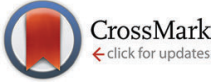

Cite this: Phys. Chem. Chem. Phys., 2015, 17, 21220

Received 31st October 2014,

Accepted 5th January 2015

DOI: $10.1039 / c 4 c p 05015 k$

www.rsc.org/pccp

\title{
Calculating average surface enhancement factors of randomly nanostructured electrodes by a combination of SERS and impedance spectroscopy $\dagger$
}

\author{
J. Kozuch, ${ }^{*^{a}}$ N. Petrusch, ${ }^{a}$ D. Gkogkou, ${ }^{a b}$ U. Gernert ${ }^{c}$ and I. M. Weidinger ${ }^{{ }^{a}}$
}

Polyhedron Ag nanostructures were created on top of a polished Au electrode via step-wise electrodeposition and tested as substrates for SERS spectroscopy. Average Raman enhancement factors were derived by combining SERS measurements with electrochemical impedance spectroscopy (EIS), which is able to determine the electroactive surface area of a randomly nanostructured surface. Depending on the deposition step an alternating increase and decrease of surface area was observed while the SERS intensity showed a clear maximum for the first deposition cycle. SEM pictures reveal the formation of Ag polyhedrons that are randomly dispersed on the Au surface. Furthermore the presence of a sub nanostructure on top of the polyhedron after the first deposition cycle is observed which becomes smoother after subsequent deposition cycles. Correlating the SEM pictures with SERS and EIS measurements it is concluded that the coral-like sub nanostructure is dominating the enhancement factor while the polyhedron structure itself only plays a minor role for electromagnetic field enhancement.

\section{Introduction}

Electromagnetic field enhancement factors (EF) created at the surface of plasmonic noble metals are used as a key parameter to judge the quality of these substrates for surface enhanced Raman spectroscopy (SERS) or surface enhanced infrared absorption (SEIRA). EF can be determined by measuring the increase in Raman or IR intensity of a molecule attached to the surface of interest in comparison to a reference system with no surface enhancement. ${ }^{1}$ As reference systems spectra of the same molecule either in solution ${ }^{2}$ or attached to a surface under "non-enhancing" conditions ${ }^{3}$ have been used in the past. The observed enhancement in signal intensity has then to be correlated to the number of molecules probed in the enhanced and in the reference system, respectively. This yields the Raman and IR enhancement factor (REF and IEF). For IR measurements IEF can be set equal to EF while for Raman measurements also the enhancement of the light scattered by the molecule has to be considered. Therefore REF is given by

\footnotetext{
${ }^{a}$ Institut für Chemie, PC 14, Technische Universität Berlin, Straße des 17. Juni 135, 10623 Berlin, Germany. E-mail: inez.weidinger@tu-berlin.de, kozuch.jacek@mailbox.tu-berlin.de

${ }^{b}$ Leibniz-Institut für Analytische Wissenschaften-ISAS-e.V., Department Berlin, Albert-Einstein-Str.9, 12489 Berlin, Germany

${ }^{c}$ Zentraleinrichtung Elektronenmikroskopie (ZELMI), Technische Universität Berlin, Straße des 17. Juni 135, 10623 Berlin, Germany

$\dagger$ Electronic supplementary information (ESI) available: SEM and EDX data of the Ag polyhedrons. See DOI: 10.1039/c4cp05015k
}

the product of $\operatorname{EF}\left(\lambda_{\text {exc }}\right)$ and $\operatorname{EF}\left(\lambda_{\text {Ra }}\right), \lambda_{\text {exc }}$ and $\lambda_{\text {Ra }}$ being the wavelength of the incident and the Raman scattered light respectively. ${ }^{4}$

The number of molecules probed on the surface is proportional to the surface area illuminated by the incident light if one assumes a constant surface molecular coverage. This makes a correct determination of the surface area so important. Unfortunately, there is no unifying rule what has to be considered as surface area. While in some work the whole illuminated surface area is used as input parameter, ${ }^{5}$ in others only the area covered with the plasmonic metal, ${ }^{2}$ and in even other cases only the area of the hot spot goes into the equation. ${ }^{6,7}$ As a result one has to be extremely careful when comparing different values of EF in the literature. These examples furthermore show that it is very important to distinguish between a local and an average EF factor. A local EF factor can only be determined for highly ordered systems that allow identification of hot spot areas. For more randomly nanostructured systems only average EF factors can be derived, as a clear assignment of areas with and without surface enhancement is not possible. Furthermore even determination of the whole illuminated surface area is extremely difficult, as it can not be derived from geometrical considerations, gained by SEM measurements. This is especially unfortunate as random nanostructures can be created very cheap and easy from wet chemistry or electrochemical techniques. ${ }^{8,9}$ For such systems, it is therefore often referred to a limit of detection (LOD), given by the minimum detectable concentration of the target molecule in solution, rather than to an enhancement factor. $^{10-12}$ LODs are often used for applied investigations in 
analytical science as here the focus is more on identifying a specific target molecule than on the characterization of the support system.

If, however, a Raman enhancement factor (REF) should be derived from a randomly nanostructured support, alternative measurements have to be performed that yield independently the surface area. One possibility is BET, which measures the amount of an inert gas physically adsorbed on the surface. However, this technique needs large surface areas to be reliable, which are not available for many nanostructures of interest. An alternative are electrochemical techniques that can be applied if the SERS support can function as an electrode. Choosing a redox active surface bound molecule, one can use cyclic voltammetry to determine the amount of molecules probed by the laser. ${ }^{13}$ In the case of redox inactive adsorbates, however, an alternative technique has to be applied.

Electrochemical impedance spectroscopy (EIS) allows determining the capacitance of the electrochemical double layer that is formed when an electrode is immersed into an electrolyte solution and ions arrange at the electrode interface. ${ }^{14}$ This double layer can be described by e.g. the Stern model, and leads to a capacitance that is directly proportional to the surface area. Thus, it can be applied to determine surface areas of randomly nanostructured electrodes using the capacitance of a perfectly flat surface as reference.

In this paper, we demonstrate how REF factors of randomly nanostructured electrodes can be derived by such a combination of SERS and EIS. Electrodes with different surface area and surface morphology were created by step-wise electrodeposition of $\mathrm{Ag}$ on top of a polished Au electrode.

\section{Experimental section}

Thiophenol, silver nitrate, sodium perchlorate, and ammonium nitrate were purchased from Sigma Aldrich. Aqueous solutions were prepared using MilliQ water $(>18 \mathrm{M} \Omega \mathrm{cm}$ ). Other chemicals and solvents were of highest purity grade available.

Electrochemical impedance measurements and deposition of silver were performed using a $\mu$ AutolabIII/FRA2 instrument and controlled using the FRA and GPES softwares. A polycrystalline gold (Au) disc electrode (R. Götze GmbH \& Co. KG) was used as working electrode, whereas a Pt-wire and $\mathrm{Ag} / \mathrm{AgCl}$ electrode $(3 \mathrm{M} \mathrm{KCl}$; non-leaking DRIREF-2 from World Precision Instruments) served as counter and reference electrodes, respectively. EIS was recorded in a $0.5 \mathrm{M} \mathrm{NaClO}_{4}$ solution at a DC potential of $-0.2 \mathrm{~V}$ using an amplitude of $25 \mathrm{mV}(\mathrm{rms})$ in a frequency range of $100 \mathrm{kHz}$ to $0.1 \mathrm{~Hz}$.

Prior to each experiment, the Au working electrode was first cleaned using acidic and basic piranha solution, then polished using Alumina polishing powders with particle sizes of ca. 1, 0.05 , and $0.02 \mu \mathrm{m}$ (Buehler) and finally cleaned electrochemically in $0.1 \mathrm{M} \mathrm{H}_{2} \mathrm{SO}_{4}$ performing 6 cyclic voltammetry cycles between 0 and $1.4 \mathrm{~V}$ at $50 \mathrm{mV} \mathrm{s}^{-1}$.

SERR spectra were acquired using the $514 \mathrm{~nm}$ line of an argon ion laser (I308, Coherent) coupled to confocal setup with a single-state spectrograph (Jobin Yvon LabRam 800 HR) equipped with a liquid-nitrogen-cooled CCD detector. The laser light was focused using a Nikon $20 \times$ objective (N. A. 0.35) with a working distance of $20 \mathrm{~mm}$. Laser power on the sample was about $1 \mathrm{~mW}$. The spectra acquisition time was $60 \mathrm{~s}$. To acquire an average spectrum of the entire surface and to avoid laser damage, the electrodes were moved constantly while measuring. To obtain a spectrum of thiophenol on a polished Au electrode, the accumulation time was increased to $120 \mathrm{~min}$. Spectra treatment was performed using an in-home programmed software. Average values and errors of the capacitance and the Raman intensities were calculated from a sum of at least 3 experiments.

SEM pictures were recorded using a Hitachi SU8030 high resolution SEM with a cold field emitter. The images were taken at $3 \mathrm{kV}$ accelerating voltage and a probe current of $60 \mathrm{pA}$. The Inlens SE and low angle BSE signals were used.

EDX measurements were carried out with an EDAX TEAM ${ }^{\mathrm{TM}}$ EDS system equipped with a $30 \mathrm{~mm}^{2}$ silicon drift detector (SDD) at excitation energy of $10 \mathrm{keV}$ and a probe current of $140 \mathrm{pA}$.

\section{Results}

Mechanically polished Au electrodes were used as a starting point. From the Au oxide reduction peak, the real area of the electrode was determined. ${ }^{15}$ For a typical polished Au electrode we found a value of $c a .1 .15 \mathrm{~cm}^{2}$ corresponding to a roughness factor of $c a$. 1.44 with a geometrical area of $0.8 \mathrm{~cm}^{2}$. These electrodes were immersed into a $2 \%(\mathrm{w} / \mathrm{w}) \mathrm{AgNO}_{3}$ solution (buffered with $20 \mathrm{mM} \mathrm{NH}_{4} \mathrm{NO}_{3}$ at $\mathrm{pH}$ 8.5) and a current of $3.3 \mathrm{~mA}$ was applied to the system for $15 \mathrm{sec}$ followed by a EIS measurement to determine the double layer capacitance. This procedure was repeated for up to 6 deposition cycles. After each cycle an SEM picture was recorded (Fig. 1), which showed a cycle dependent Ag surface nanostructure on top of the Au electrode.

After the first deposition cycle, formation of sporadic $\mathrm{Ag}$ nano-polyhedrons with triangular lateral faces is observed in the SEM. The average diameter of the polyhedrons $d_{\mathrm{PH}}$ was determined by taking the longest length of its cross section as demonstrated in Fig. S2 (ESI $\dagger$ ). The so derived values for $d_{\mathrm{PH}}$ vary between 2.6 and $4.4 \mu \mathrm{m}( \pm 15 \%)$ depending on the deposition cycle (see Table 1). At higher resolution it can be seen, that each polyhedron exhibits a smaller coral-like sub nanostructure. Subsequent deposition cycles lead to an increase of the polyhedron surface coverage on the Au support. Furthermore, it can be seen that the sub nanostructure becomes smoother. After the 6th deposition cycle no significant sub nanostructure can be seen anymore leading to a polyhedron structure with sharp edges and smooth sides. EDX spectra confirm that the polyhedrons consist of $\mathrm{Ag}$ and not an $\mathrm{Ag}-\mathrm{Au}$ alloy (see Fig. S1, ESI $\dagger$ ).

After each deposition cycle, an impedance spectrum was recorded. From the frequency-weighted Cole-Cole plot (Fig. 2), the double layer capacitance $C$ of the electrodes was derived directly by reading out the diameter of the half-circle. ${ }^{16}$ This approach was already used previously to determine the capacitance value of self-assembled monolayers and lipid bilayers, and provides the advantage that no model-dependent fit has to be applied to the data. $^{16,17}$ 


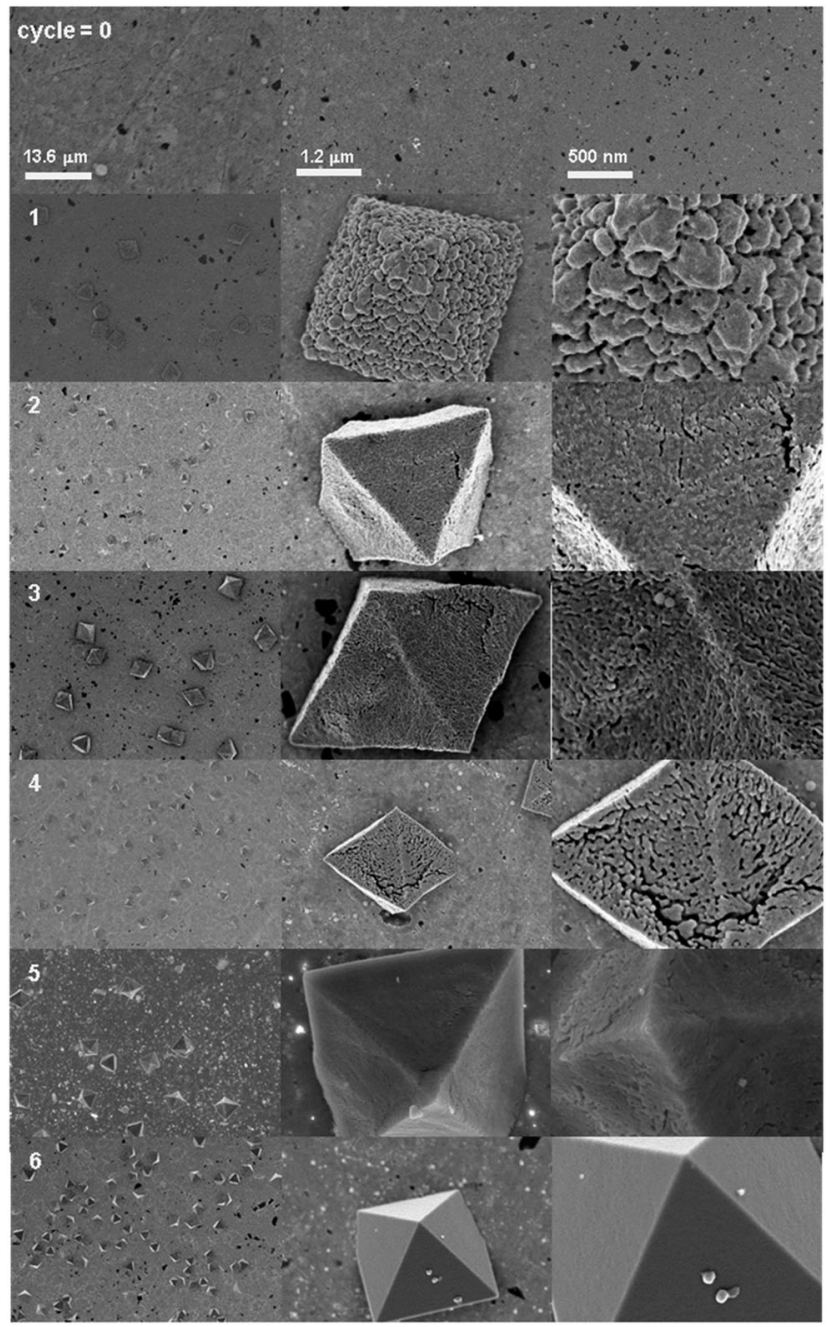

Fig. 1 From top to bottom: SEM pictures of AgaAu electrodes after 0, 1 , $2,3,4,5$, and 6 deposition cycles with different spatial resolution.

Table 1 Average polyhedron diameter $\left(d_{\mathrm{PH}}\right)$, capacitance $(C)$, SERS intensity (ISERS), illuminated surface area ( $A$ ) and Raman enhancement factors (REF) for different deposition cycles

\begin{tabular}{llrrrlr}
\hline Cycle & $d_{\mathrm{PH}} / \mu \mathrm{m}$ & \multicolumn{1}{c}{$C / \mu \mathrm{F}$} & \multicolumn{1}{c}{$I_{\mathrm{SERS}} / \mathrm{cts} \mathrm{mW}^{-1} \mathrm{~s}^{-1}$} & \multicolumn{1}{c}{$A / \mu \mathrm{m}^{2}$} & $\mathrm{REF} 1$ & $\mathrm{REF} 2$ \\
\hline 0 & - & 27.40 & 0.33 & 18.1 & $1.1 \times 10^{2}$ & 1 \\
1 & 4.3 & 196.82 & 2242.47 & 130.0 & $1.0 \times 10^{5}$ & 946.0 \\
2 & 3.3 & 103.10 & 586.13 & 68.1 & $5.1 \times 10^{4}$ & 472.0 \\
3 & 4.4 & 195.65 & 92.10 & 129.1 & $4.2 \times 10^{3}$ & 39.1 \\
4 & 3.8 & 135.70 & 62.55 & 89.6 & $4.1 \times 10^{3}$ & 38.3 \\
5 & 4.1 & 154.05 & 239.05 & 101.7 & $1.4 \times 10^{4}$ & 128.8 \\
6 & 2.6 & 49.00 & 156.75 & 32.3 & $2.9 \times 10^{4}$ & 265.6
\end{tabular}

Here, for a bare electrode surface, the capacitance arises from the formation of the Helmholtz layer of adsorbed ions and the diffuse layer of electrostatically attracted species. Thus, it results from the combination of the Helmholtz capacitance $C_{\mathrm{H}}$ and the diffuse capacitance $C_{\mathrm{D}}:^{14,18}$

$$
\frac{1}{C}=\frac{1}{C_{\mathrm{H}}}+\frac{1}{C_{\mathrm{D}}}
$$

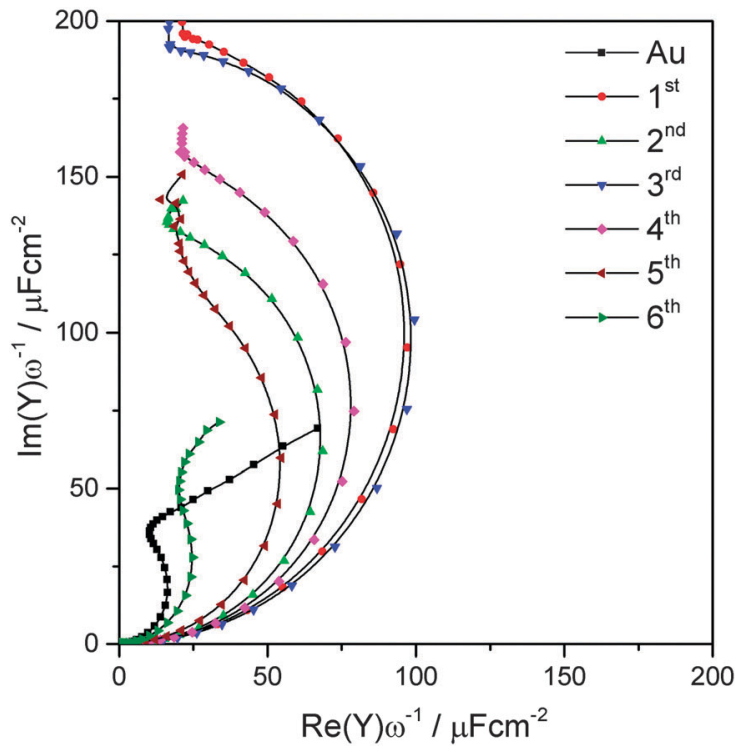

Fig. 2 Frequency-weighted Cole-Cole plots of the impedance spectra of the pure $\mathrm{Au}$ electrode and the Ag@Au electrodes after each deposition cycle. The lines connect the points to guide the eye.

At high electrolyte concentrations $(>0.1 \mathrm{M})$, the diffuse layer decreases $(<10 \mathrm{~nm})$ and its capacitance assumes a rather high value. Consequently, the total capacitance is dominated by the Helmholtz capacitance ${ }^{19,20}$

$$
C \approx C_{\mathrm{H}}=\varepsilon_{0} \varepsilon_{\mathrm{r}} \frac{A}{d_{\mathrm{H}}}
$$

where $\varepsilon_{0}$ and $\varepsilon_{\mathrm{r}}$ are the absolute and relative permittivites, $d_{\mathrm{H}}$ the thickness of the Helmholtz layer, and $A$ the surface area of the electrode. For noble metals, e.g. $\mathrm{Au}$ and $\mathrm{Ag}$, double layer capacitance values of $c a .10-40 \mu \mathrm{F} \mathrm{cm} \mathrm{cm}^{-2}$ are reported in the literature. ${ }^{14,19-22}$ To avoid specific binding of the electrolyte ions to the electrode surface, it is common practice to use a weakly interacting electrolyte, such as $\mathrm{NaClO}_{4} \cdot{ }^{21,22}$ Therefore, we employed a $0.5 \mathrm{M} \mathrm{NaClO}_{4}$ solution for the impedance measurements. Here, we observed for mechanically polished $\mathrm{Au}$ surfaces an absolute capacitance value of $27.4 \mu \mathrm{F}$. As evident from eqn (2), the increase in $C$ can be directly transformed into an increase in surface area (Fig. 4A). Interestingly not a steady, but an alternating increase of surface area is observed as a function of deposition cycle. This alteration is very strong for the first cycles and smoothers out for the latter ones. After 6 deposition cycles the capacitance is almost back to its initial value. This indicates that not the formation of the polyhedrons itself, but the change of the sub nanostructure on top is responsible for the increase in surface area.

The observed large changes in surface area resulting from different surface roughness underline the difficulties to determine real surface areas from SEM pictures. Especially comparing cycles 1 and 3 with cycle 6 it can be seen that the real surface area determined from EIS in 1 and 3 exceeds the one of 6 by a factor of 4 albeit the surface coverage of the Ag polyhedrons itself is increased in 6 judging from the SEM pictures in Fig. 1. 


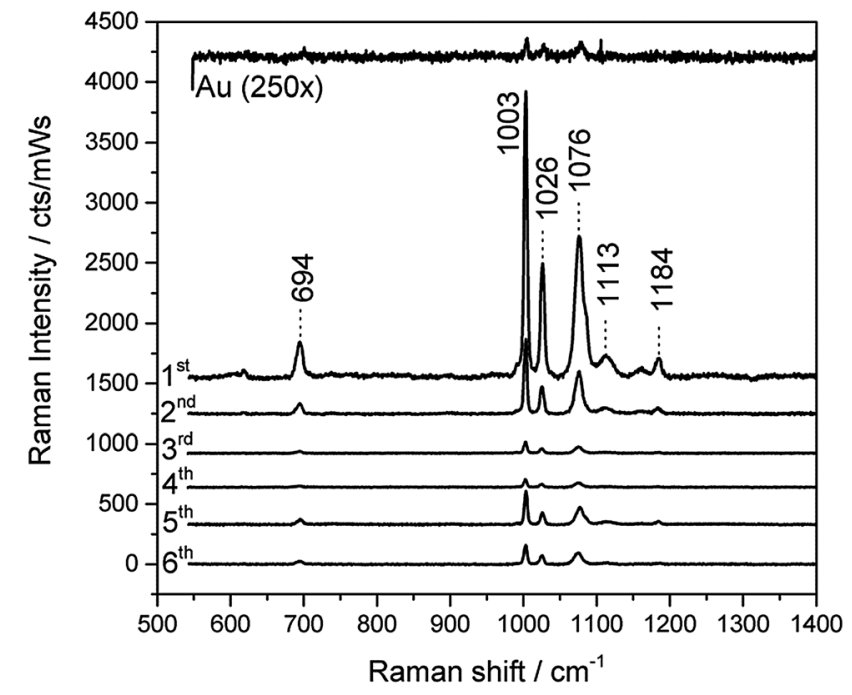

Fig. 3 SERS intensity of thiophenol attached to Ag@Au electrodes for different deposition cycles.

The electrodes, prepared with increasing deposition steps, were incubated separately in an ethanolic solution of $1 \mathrm{mM}$ thiophenol (TP) for $30 \mathrm{~min}$, washed, dried and measured with SERS. The SER spectra shown in Fig. 3 give the typical vibrational pattern of TP. The Au electrode alone yields an almost non-detectable Raman signal, which is reasonable as the polished Au electrode is not expected to exhibit any electromagnetic surface enhancement using $514 \mathrm{~nm}$ laser excitation. The symmetric stretching vibration of the phenyl ring at $1003 \mathrm{~cm}^{-1}$ is used in the following to determine SERS intensity of the respective electrodes (see Fig. 4B). The SERS intensity did not show the same dependence on deposition cycle as the surface area. Here a clear maximum can be seen for the first cycle with a subsequent decrease up to the 4 th cycle, from which on an increase in SERS is seen again.

\section{Determination of absolute surface area}

In a first step it was checked whether the real surface area derived from impedance measurements corresponds to the values obtained by other methods such as cyclic voltammetry ${ }^{22}$ or BET. ${ }^{5}$ This was done for polished and roughened $\mathrm{Au}$ and $\mathrm{Ag}$ electrodes respectively. Plotting the roughness factors obtained by EIS against the values derived from the reference measurements yielded a straight line with a slope of 1.07 (Fig. S3, ESI†). In a previous work Hupp et al. showed similar results correlating the roughness factor of pure $\mathrm{Ag}$ electrodes with its capacitance values. ${ }^{21}$ Thus we can safely say that EIS gives reliable values for the real surface area of a nanostructured electrode with roughness factors below 20. Furthermore dividing the measured capacitance of the polished $\mathrm{Au}$ electrode by its real surface area $1.15 \mathrm{~cm}^{2}$, we obtain a specific capacitance value of $23.8 \mu \mathrm{F} \mathrm{cm}^{-2}$, which is roughly in line with the previously determined capacitances of 22.9 and $22 \mu \mathrm{F} \mathrm{cm} \mathrm{cm}^{-2}$ for a mono- and polycrystalline $\mathrm{Au}$ electrode respectively. ${ }^{23,24}$

The area illuminated by the laser can be derived by multiplying the area of the laser spot with the roughness factor of the electrode. The focus of the laser at $514 \mathrm{~nm}$ using a $20 \times$ objective has been determined previously to be $2 \mu \mathrm{m}^{2,5}$ resulting in an illuminated
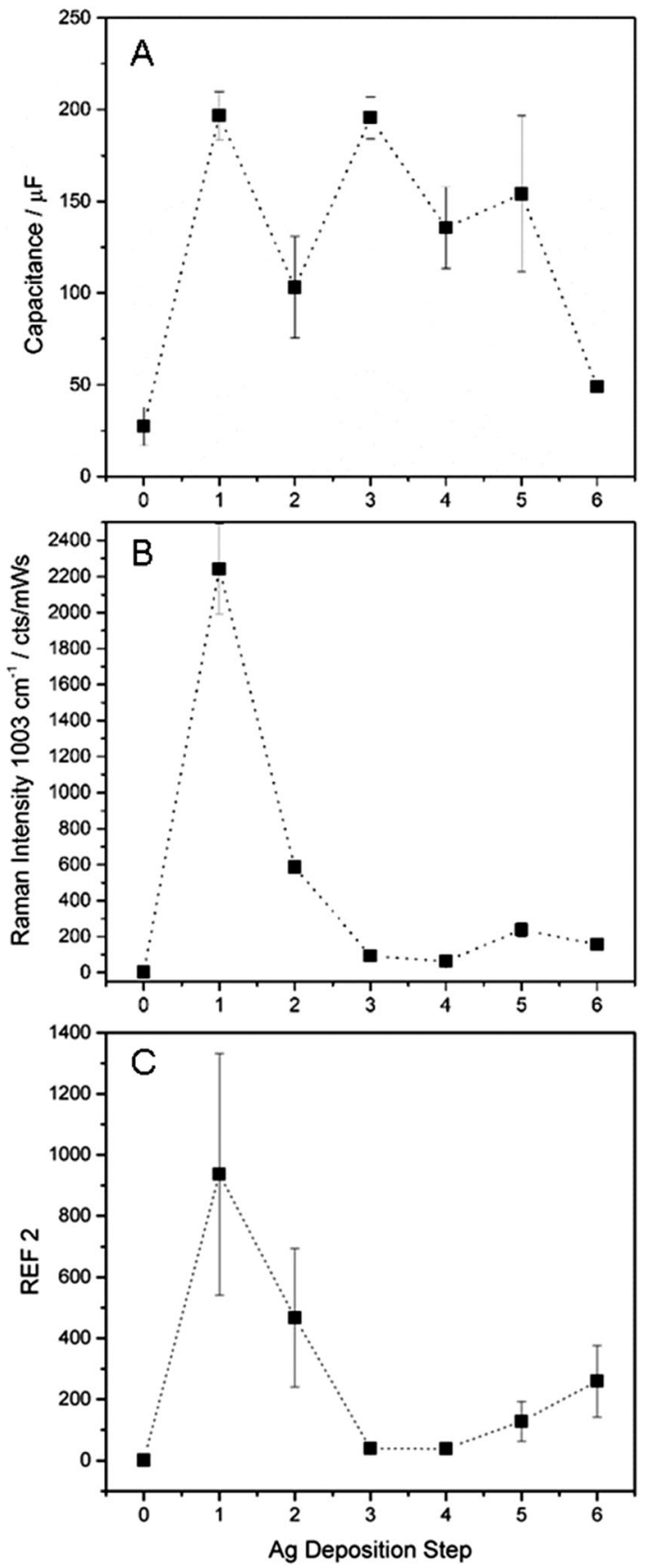

Fig. 4 From top to bottom: capacitance, SERS intensity (errors for the intensity were in the range of $10-15 \%$ ) and REF 2 as a function of deposition cycle. The errors for REF 2 were determined using the propagation of uncertainty.

area of $A_{0}=18.1 \mu \mathrm{m}^{2}$. Using this value as reference for the $\mathrm{Au}$ electrode prior to $\mathrm{Ag}$ deposition, the surface area of the nanostructured Ag@Au electrodes ( $\left.A_{\text {SERS }}\right)$ can be calculated by:

$$
A_{\mathrm{SERS}}=A_{0} \frac{C_{\mathrm{SERS}}}{C_{0}}
$$

$C_{\text {SERS }}$ and $C_{0}$ being the capacitances of the SERS and reference system, respectively. The so derived surface areas are summarized in Table 1. 


\section{Calculation of REF}

The general equation for determining REF is given by:

$$
\mathrm{REF}=\frac{I_{\text {SERS }}}{I_{0}} \cdot \frac{N_{0}}{N_{\text {SERS }}}
$$

where $I_{0}$ and $N_{0}$ refer to the Raman intensity and number of molecules probed in a reference system with no field enhancement $(\mathrm{REF}=1)$. The reference system can be either a Raman measurement of the probe molecules in solution (1), adsorbed on a "non-enhancing" surface (2) or on the same system illuminated with perpendicular polarized light (3). As option 3 requires highly ordered support symmetry only the options 1 and 2 will be considered. This leads to the following derivation of eqn (4):

$$
\mathrm{REF} 1=\frac{I_{\mathrm{SERS}}}{I_{0}} \cdot \frac{c_{0} \cdot V_{0}}{\Gamma \cdot A_{\mathrm{SERS}}}
$$

and

$$
\mathrm{REF} 2=\frac{I_{\mathrm{SERS}}}{I_{0}} \cdot \frac{\Gamma \cdot A_{0}}{\Gamma \cdot A_{\mathrm{SERS}}}=\frac{I_{\mathrm{SERS}}}{I_{0}} \cdot \frac{C_{0}}{C_{\mathrm{SERS}}}
$$

$A_{0}, A_{\text {SERS }}$ and $V_{0}$ belong to the area and volume illuminated by the laser. $\Gamma$ stands for the average surface coverage of the molecule, which was determined previously for TP to be $1.1 \mathrm{nmol} \mathrm{cm}{ }^{-2}$.

$V_{0}$ can be determined from the radius of the laser spot $(r=2 \mu \mathrm{m})$ if one approximates the illuminated volume of a Gaussian-shaped beam by a cylinder with the volume: ${ }^{5}$

$$
V_{0}=\frac{2 \pi^{2} r^{4}}{\lambda}
$$

The biggest error in option 1 comes from an inaccurate estimation of $V_{0}$ which most likely leads to an overestimation of REF. The biggest error in option 2 on the other hand comes from the assumption that the reference system exhibits no surface enhancement, which generally leads to an underestimation of REF.

REF values derived from both approaches are summarized in Table 1 and for REF 2 in Fig. 4C. For determination of REF 1, $I_{0}=90 \mathrm{cts} \mathrm{mW}^{-1} \mathrm{~s}^{-1}$ and $c_{0}=9.54 \mathrm{M}$ were taken from a previous work; ${ }^{5}$ for REF $2, A_{\text {SERS }}$ and $A_{0}$ were determined as shown above and listed in Table 1.

Depending on the approach, values of REF are obtained that differ by roughly 2 orders of magnitude. The sole reason for this discrepancy is the REF value obtained for the pure Au electrode, which is set to 1 in option 2 but determined to be 110 in option 1.

In principle flat $\mathrm{Au}$ electrodes should not give any plasmon induced electromagnetic enhancement at $514 \mathrm{~nm}$ excitation. The fact that REF 1 is nevertheless larger than one can have different reasons: first, the illuminated volume $V_{0}$ in eqn (5) is in reality smaller than assumed. Second, a small contribution of chemical enhancement cannot be ruled out. Third, solution measurements probe molecules with an isotropic orientation whereas SERS monitors TP in a well-defined orientation in respect to the surface. Last but not least the roughness factor of the pure Au electrode is larger than one. Therefore electric field enhancement due to multiple scattering of light as observed for non plasmonic but nanostructured surfaces ${ }^{25}$ might take place. All these considerations might sum up to the observed 2 order of magnitude difference between REF 1 and 2. Both values for REF 1 and REF 2 have therefore to be seen as an upper and lower limit for the real REF. Nevertheless, it becomes clear that the definition of the reference system gives the biggest scattering in the values of REF.

The relative surface enhancement as a function of deposition cycle on the other hand can be determined quite precisely with this method. It is interesting to note that the highest enhancement is achieved for the first cycle albeit the surface area changes in an alternating manner.

After the 1st deposition cycle a very rough sub nanostructure is observed in the SEM picture. An explanation for this might be that on Au supports nucleation and growth of $\mathrm{Ag}$ seeds is favored in contrast to formation of a thin $\mathrm{Ag}$ film. This hypothesis is supported by the fact that the same experiments using a flat $\mathrm{Ag}$ electrode as support did not show this high surface area and alternating behavior (data not shown).

Even if a detailed explanation of the underlying electrodeposition mechanism goes beyond the scope of this work, we speculate that the second deposition cycle fills up the gaps between the Ag corals. With the 3rd cycle a second Ag layer may be formed on top of the existing one and it seems from the SEM data that the polyhedron surface coverage on the Au support is increased. These processes combined may lead to the alternating decrease and increase in surface area observed in Fig. 4A. The surface enhancement on the other hand shows a clear maximum for the 1st deposition cycle and a minimum for the 3rd and 4th cycle. REF is therefore not connected to the overall surface area, which is the same for the 1st and 3rd deposition cycle, but to the morphology of the sub nanostructure itself. Especially the presence of small gaps between the coral like nanostructure, seen in the surface structure after the 1st deposition cycle, might cause the formation of hot spots with very high local field enhancement. From the 4 th cycle on, a small but steady increase in REF is observed. SEM pictures recorded after the 6th cycle do show that the polyhedron surface coverage has been increased but the sub nanostructure of individual polyhedrons has been smoothed out significantly. Still the gap between two neighbored polyhedrons is too large to assume that hot spots are formed inside this gap. Therefore we assume that the biggest contribution of the surface enhancement is given in this case by the sharp edges of individual polyhedrons.

\section{Conclusions}

Stepwise electrodeposition of $\mathrm{Ag}$ on polished $\mathrm{Au}$ electrodes resulted in the formation of polyhedron nanostructures that where randomly spread over the Au surface. Depending on the deposition step a sub nanostructure was formed and smoothed out on the polyhedron surface leading to an alternating overall surface area.

EIS spectroscopy of the electrical double layer made it possible to determine the surface area of these randomly nanostructured 
electrodes. The SERS intensity of a TP SAM adsorbed on the Ag@Au electrodes was measured and corrected for the different surface areas yielding the respective Raman enhancement factor for each electrode as a function of deposition cycle. The biggest contribution to the surface enhancement came from sub nanostructure whereas the polyhedron nanostructure itself only played a minor role as plasmonic amplifier.

The absolute values for REF differed by roughly 2 orders of magnitude depending on the reference system chosen. This underlines the problems when comparing absolute values for electromagnetic surface enhancement of different support systems in the literature.

\section{Acknowledgements}

The authors would like to thank Uwe Kuhlmann, Diego Millo, Khoa Ly and Peter Hildebrandt for valuable support. Financial support by the DFG (Unicat, SALSA, INST 131/631-1) is greatly acknowledged.

\section{Notes and references}

1 E. C. Le Ru and P. G. Etchegoin, Principles of SurfaceEnhanced Raman Spectroscopy and Related Plasmonic Effects, Elsevier, 2009.

2 A. D. McFarland, M. A. Young, J. A. Dieringer and R. P. Van Duyne, J. Phys. Chem. B, 2005, 109, 11279-11285.

3 B. Fazio, C. D’Andrea, F. Bonaccorso, A. Irrera, G. Calogero, C. Vasi, P. G. Gucciardi, M. Allegrini, A. Toma, D. Chiappe, C. Martella and F. Buatier De Mongeot, ACS Nano, 2011, 5, 5945-5956.

4 F. Siebert and P. Hildebrandt, Vibrational Spectroscopy in Life Science, Wiley-VCH, 2008.

5 H. K. Ly, C. Köhler, A. Fischer, J. Kabuss, F. Schlosser, M. Schoth, A. Knorr and I. M. Weidinger, Langmuir, 2012, 28, 5819-5825.

6 L. Rodríguez-Lorenzo, R. A. Álvarez-Puebla, I. Pastoriza-Santos, S. Mazzucco, O. Stéphan, M. Kociak, L. M. Liz-Marzán and F. J. G. De Abajo, J. Am. Chem. Soc., 2009, 131, 4616-4618.

7 C. Huck, F. Neubrech, J. Vogt, A. Toma, D. Gerbert, J. Katzmann, T. Härtling and A. Pucci, ACS Nano, 2014, 8, 4908-4914.
8 Z. Q. Tian, B. Ren and D. Y. Wu, J. Phys. Chem. B, 2002, 106, 9463-9483.

9 P. C. Lee and D. Meisel, J. Phys. Chem., 1982, 86, 3391-3395.

10 X. X. Han, A. M. Schmidt, G. Marten, A. Fischer, I. M. Weidinger and P. Hildebrandt, ACS Nano, 2013, 7, 3212-3220.

11 X. X. Han, Y. Kitahama, T. Itoh, C. X. Wang, B. Zhao and Y. Ozaki, Anal. Chem., 2009, 81, 3350-3355.

12 P. Negri, K. T. Jacobs, O. O. Dada and Z. D. Schultz, Anal. Chem., 2013, 85, 10159-10166.

13 M. Sezer, R. Spricigo, T. Utesch, D. Millo, S. Leimkuehler, M. A. Mroginski, U. Wollenberger, P. Hildebrandt and I. M. Weidinger, Phys. Chem. Chem. Phys., 2010, 12, 7894-7903.

14 V. F. Lvovich, Impedance Spectroscopy: Applications to Electrochemical and Dielectric Phenomena, John Wiley \& Sons, New York, 2012.

15 S. Trasatti and O. A. Petrii, Pure Appl. Chem., 1991, 63, 711-734.

16 L. J. C. Jeuken, N. N. Daskalakis, X. Han, K. Sheikh, A. Erbe, R. J. Bushby and S. D. Evans, Sens. Actuators, B, 2007, 124, 501-509.

17 J. Kozuch, C. Weichbrodt, D. Millo, K. Giller, S. Becker, P. Hildebrandt and C. Steinem, Phys. Chem. Chem. Phys., 2014, 16, 9546-9555.

18 V. Lockett, M. Horne, R. Sedev, T. Rodopoulos and J. Ralston, Phys. Chem. Chem. Phys., 2010, 12, 12499-12512.

19 A. Wieckowski, Interfacial Electrochemistry: Theory, Experiment and Application, Marcel Dekker Inc., New York, 1999.

20 A. J. Bard and L. R. Faulkner, Electrochemical Methods: Fundamentals and Application, John Wiley \& Sons, New York, 2nd edn, 2001.

21 J. T. Hupp, D. Larkin and M. J. Weaver, Surf. Sci., 1983, 125, 429-451.

22 M. E. Abdelsalam, P. N. Bartlett, J. J. Baumberg, S. Cintra, T. A. Kelf and A. E. Russell, Electrochem. Commun., 2005, 7, 740-744.

23 R. Janek, W. Fawcett and A. Ulman, J. Phys. Chem. B, 1997, 101, 8550-8558.

24 U. Oesch and J. Janata, Electrochim. Acta, 1983, 28, 1237-1246.

25 X. X. Han, C. Köhler, J. Kozuch, U. Kuhlmann, L. Paasche, A. Sivanesan, I. M. Weidinger and P. Hildebrandt, Small, 2013, 9, 4175-4181. 